\title{
Change of parameters in molecular structure of Donbas coals under the influence of external factors
}

\author{
Liudmyla Pymonenko,", Oleksandr Burchak ${ }^{1}$, Oleksandr Balalaiev ${ }^{1}$, Valentyna \\ Slobodiannykova ${ }^{1}$, and Yurii Sierikov ${ }^{1}$ \\ ${ }^{1}$ Institute of Geotechnical Mechanics named by N. Poljakov of National Academy of Sciences of \\ Ukraine, 49005, Dnipro, Simferopolska Str., 2a, Ukraine
}

\begin{abstract}
The molecular structure of coal is estimated by 13 parameters of infrared spectra, electron paramagnetic resonance, volatile yield and ash content. A new criterion of hydrophobicity is applied, which has shown its informativeness in analyzing the molecular structure of coals of various ranks. It is more sensitive to changes in coal rank than the standard index of volatile yield. The coalification process leads to a significant increase in this index due to the water release and other hydroxyl-containing compounds from the substance of coal. Two-way analysis of variance showed that the influence of the metamorphism factor is significant for 9 molecular parameters. The strongest effects are manifested in the sorption capacity, the criterion of hydrophobicity and the number of paramagnetic centers. The stratigraphic factor does not have a significant impact on one of the molecular parameters. Factor analysis by the method of principal components of the molecular structure of coal showed that the most significant independent factors are metamorphism and sedimentation conditions, the latter ones include independent processes: accumulation of mineral components, decomposition of biomass and geochemical environment during the sedimentation period.
\end{abstract}

\section{Introduction}

The complexity of the current economic situation in Ukraine, to a large extent, is due to a shortage of energy resources, although there is about 56.7 billion tons of coal in the depths of the main coal-bearing basins; and methane resources in the coal-bearing stratum, according to assessments of various researchers, are 12-25 trillion $\mathrm{m}^{3}$. But most of the coals upon their qualities cannot be used neither in energy nor coke chemistry. The method of artificially transferring coals to a higher degree of metamophism is absent, which is due to both the complexity of the object of research and the ambiguity of ideas about the processes that occur in the molecular structure of coals under the influence of natural factors. Indeed, during the accumulation of sediments, which are about 300 million years, thermodynamic, tectonic, chemical, biochemical and many other factors changed significantly, each of

*Corresponding author: gvrvg@meta.ua 
which made its own contribution to the formation of the coal structure.

Currently, there are a fairly large number of theoretical and experimental studies on the structure and state of coal by various physical, physicochemical and chemical methods [1]. However, in recent years, the discrepancy between the results of geological and geochemical studies of coalification processes with theoretical calculations and with the results of simulation of these processes in the laboratory has been very often observed. Given the complexity and multifactorial nature of external processes, researchers, as a rule, analyze in detail the change in one of the factors. Therefore, the study of the cumulative effect in a number of external factors and the selection of the main one, formed the modern structure of coal, is relevant and is a necessary link in the research of the formation of coalgas deposits.

\section{Methodology}

The molecular structure of coal was estimated on the basis of three measurement methods, the results of which were used to determine groups of indicators.

Technical analysis of coal is ash content and volatile yield.

Electron Paramagnetic Resonance (EPR) spectroscopy makes it possible to determine the number of paramagnetic centers (PMC), the width of the EPR signal, the coefficient of aromatization, Sorption capacity and conjugacy coefficient.

Diffuse Reflection Infrared Fourier Transformation (DRIFT) allows us to find the length of aliphatic chains (the ratio of the number of methylene and methyl groups), the vibrational mode of $\mathrm{CH}$ groups (the ratio of asymmetric and symmetric valence vibrations), the band width of the valence vibrations in the aliphatic groups (the ratio of the total height and width of the model Gaussian peaks). This method also determined the ratio of the number of protons attached to aromatic rings and aliphatic chains, the criterion of hydrophobicity (the ratio of different $\mathrm{CH}$ to various $\mathrm{OH}$ groups) and the content of carbonyl groups in organic compounds.

Most of these indexes are often used to characterize the substance of coal [2-4]. In addition, a new criterion for the hydrophobicity of undisturbed coals is introduced here. In this case, the coals are not subjected to acid leaching, [5]. In the short-wave part of the average standard range of DRIFT $\left(2000-3800 \mathrm{~cm}^{-1}\right)$ of organic coal substance, the valence vibrations of protons relative to heavier atoms $(\mathrm{O}, \mathrm{C}, \mathrm{N}, \mathrm{S}, \mathrm{P}$, etc.) lie. These bands have different frequencies and characteristic peaks shape, which allows us to identify and separate the vibrations of $\mathrm{CH}$ and $\mathrm{OH}$ of the functional groups. The series of sharp peaks shaded with a black background (Fig. 1) corresponds to aliphatic and aromatic $\mathrm{CH}$ groups. A wide diffuse asymmetric peak with a gray background belongs to the valence vibrations of hydroxyls. Free vibrations are the left steeply dipping wing and involved in numerous hydrogen bonds - the right smoothly dipping wing. The stronger the intermolecular interaction the lower the frequency of hydroxyl.

The criterion of hydrophobicity is calculated as the ratio of the areas of black and gray figures. Its chemical meaning lies in the fact that $\mathrm{CH}$ groups of organic compounds have hydrophobic properties, and $\mathrm{OH}$ groups of organic and mineral substances are hydrophilic ones. A wide hydroxyl band includes not only vibrations of hydroxides, alcohols, acids and other compounds containing hydroxogroups, but also vibrations of crystallization or constitutional water set in the molecular structure of coal.

The advantages of this index include a good recognition of the used groups of bands in coals of varying degrees of metamorphism. This allows conducting a reliable assessment of the hydrophobicity criterion for all grades of coal.

Coals were selected for the research: lower (beds $\left.\mathrm{c}_{1}-\mathrm{c}_{10}\right)$, medium $\left(\mathrm{k}, 1, \mathrm{~m}_{3}\right)$ and upper carbon $\left(\mathrm{n}_{1}\right)$, differing in sedimentation conditions, the degree of metamorphism of 
precipitation accumulation time, which made it possible to research the influence of these factors on the coal material.

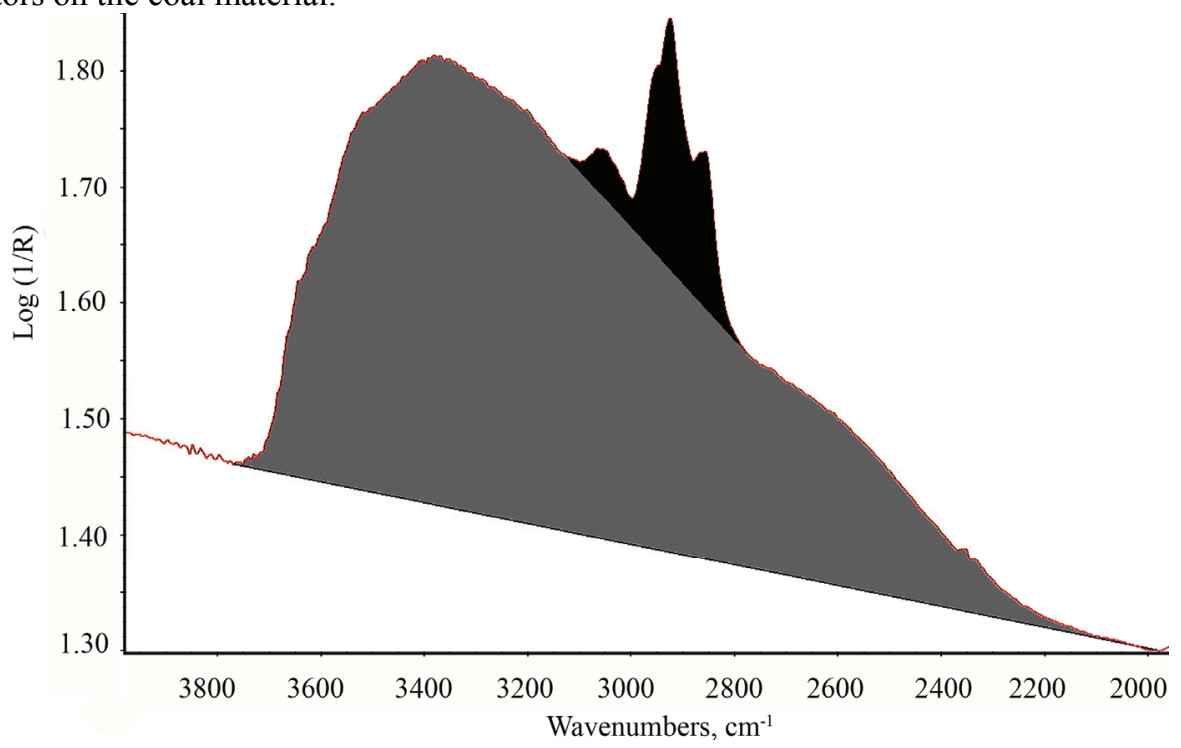

Fig. 1. Fragment of typical DRIFT for sub-bituminous coal with marked areas of vibrational bands, specific $\mathrm{CH}$ (dark background), $\mathrm{OH}$ (gray background) functional groups ( $\mathrm{R}$ - reflectivity).

\section{Results and their Discussion}

Conducted two-factor analysis of variance of the entire massif of molecular parameters of coal showed that the five-level (coal beds c, k, 1, m, n) stratigraphic factor (Bed) did not significantly affect any of the responses (Table 1). The three-level: Sub-bituminous (SB), High volatile bituminous (HVB) and Low volatile bituminous (LVB) metamorphism factor (Rank) reliably affects most of the researched coal parameters.

Table 1. Descriptive statistics and Fisher criteria for molecular parameters of coal with influence effects of metamorphism and stratigraphy factors.

\begin{tabular}{|l|c|c|c|c|c|}
\hline \multirow{2}{*}{\multicolumn{1}{|c|}{ Responces }} & \multicolumn{2}{c|}{ Rank factor levels } & \multicolumn{2}{c|}{ Factors } \\
\cline { 2 - 6 } & SB & HVB & LVB & Rank & Bed \\
\hline Ash content & $5.77 \pm 6.04$ & $10.33 \pm 7.1$ & $10.5 \pm 5.97$ & 0.31 & 2.87 \\
\hline Volatile yield & $40.07 \pm 4.1$ & $28.91 \pm 4.2$ & $16.47 \pm 5.6$ & $\mathbf{1 1 . 8 4}$ & 0.04 \\
\hline PMC quantity & $2.00 \pm 0.53$ & $2.91 \pm 0.45$ & $3.94 \pm 0.46$ & $\mathbf{1 6 . 0 5}$ & 0.41 \\
\hline ESR signal width & $6.32 \pm 0.59$ & $6.31 \pm 0.90$ & $6.35 \pm 0.36$ & 0.38 & 1.01 \\
\hline Aromatization coefficient & $0.37 \pm 0.09$ & $0.51 \pm 0.13$ & $0.75 \pm 0.07$ & $\mathbf{1 5 . 2 6}$ & 1.22 \\
\hline Sorption capacity & $9.26 \pm 3.89$ & $18.50 \pm 6.9$ & $35.68 \pm 3.0$ & $\mathbf{2 4 . 7 6}$ & 0.02 \\
\hline Contingency coefficient & $0.62 \pm 0.24$ & $1.34 \pm 0.77$ & $3.28 \pm 1.02$ & $\mathbf{1 4 . 4 7}$ & 0.89 \\
\hline Aliphatic chain length & $0.38 \pm 0.25$ & $0.24 \pm 0.08$ & $0.19 \pm 0.12$ & $\mathbf{1 0 . 3 3}$ & 0.22 \\
\hline Oscillation mode of CH groups & $2.40 \pm 0.56$ & $1.90 \pm 0.46$ & $1.78 \pm 0.22$ & 0.10 & 0.58 \\
\hline Peak width of CH groups & $0.31 \pm 0.06$ & $0.28 \pm 0.06$ & $0.22 \pm 0.06$ & 1.30 & 1.11 \\
\hline Aromatic of protons & $0.14 \pm 0.09$ & $0.49 \pm 0.22$ & $0.79 \pm 0.1$ & $\mathbf{9 . 9 9}$ & 2.52 \\
\hline Criterion of hydrophobicity & $0.06 \pm 0.02$ & $0.10 \pm 0.03$ & $0.17 \pm 0.03$ & $\mathbf{1 8 . 5 9}$ & 1.80 \\
\hline Carbonyl groups & $2.95 \pm 2.08$ & $0.65 \pm 1.01$ & $0.11 \pm 0.05$ & $\mathbf{8 . 1 6}$ & 1.17 \\
\hline
\end{tabular}

Note: Fisher's criteria for accurate effects at a significance level of $\mathrm{p}<0.05$ are marked in bold type.

The strongest effects were recorded for: sorption capacity -24.76 ; hydrophobicity 
criterion -18.59 ; the number of PMC - 16.05; the aromatization coefficients -15.26 and conjugacy one -14.47 ; volatile yield -11.4 , etc. The analysis of the simultaneous influence of these two factors with a reduced number of beds for the full plan of the experiment showed the absence of accurate effects for all dependent variables. Thus, of the two factors considered, the metamorphism of coals in the process of their immersion is the most significant and accurate factor. The total Fisher criterion for all responses $(F=3.99)$ is more than 2.5 times greater than that of the stratigraphic factor.

The factor analysis by the principal component method (Principal Components Analysis (PCA)) allows one to identify and rank upon importance the independent latent factors affecting all the indicators under research. On the basis of the Kaiser criterion, a model of 4 main components (PCA) was chosen, which explains $85 \%$ of the total variance of standardized features (Table 2).

$56.8 \%$ of dispersion accounts at the first most significant factor. It strongly affects most of the features and is caused by the process of coal metamorphism. The obtained result is closely correlated with the results of dispersion analysis. The second $(11.4 \%)$, third $(8.9 \%)$ and fourth $(7.8 \%)$ factors affect the variability of the ash content, the length of aliphatic chains and the width of the EPR signal, respectively. It is obvious that these factors are independent of each other, and their action is maximal during the period of sedimentation.

Table 2. Factor loads on the features of the molecular structure of coal.

\begin{tabular}{|l|c|c|c|c|}
\hline Features & PCA1 & PCA2 & PCA3 & PCA4 \\
\hline Ash content & 0.05 & $\mathbf{0 . 9 1}$ & -0.04 & 0.03 \\
\hline Volatile yield & $\mathbf{- 0 . 9 2}$ & -0.22 & 0.03 & 0.10 \\
\hline PMC quantity & $\mathbf{0 . 9 2}$ & 0.10 & 0.02 & -0.14 \\
\hline ESR signal width & -0.10 & 0.06 & 0.12 & $\mathbf{- 0 . 9 3}$ \\
\hline Aromatization coefficient & $\mathbf{0 . 9 5}$ & 0.17 & 0.13 & 0.12 \\
\hline Sorption capacity & $\mathbf{0 . 9 6}$ & 0.14 & 0.10 & 0.03 \\
\hline Contingency coefficient & $\mathbf{0 . 8 9}$ & 0.13 & 0.23 & 0.17 \\
\hline Aliphatic chain length & -0.30 & -0.22 & $\mathbf{- 0 . 7 8}$ & 0.21 \\
\hline Oscillation mode of CH groups & -0.58 & -0.32 & 0.56 & 0.11 \\
\hline Peak width of CH groups & -0.29 & -0.69 & -0.17 & 0.14 \\
\hline Aromatic of protons & $\mathbf{0 . 8 5}$ & 0.46 & -0.10 & 0.01 \\
\hline Criterion of hydrophobicity & $\mathbf{0 . 8 7}$ & 0.04 & 0.09 & 0.30 \\
\hline Carbonyl groups & $\mathbf{- 0 . 7 4}$ & -0.16 & 0.32 & 0.34 \\
\hline Eigenvalues & 7.39 & 1.49 & 1.16 & 1.02 \\
\hline Total dispersion proportion, \% & 56.83 & 11.43 & 8.92 & 7.84 \\
\hline
\end{tabular}

Note: significant loads of less than -0.7 and more than 0.7 are marked in bold type.

The accumulation of mineral components in coal is mainly determined by the river runoff, groundwater or sea tides. The ash components of coal molecular structure are in the form of inorganic ions of soluble salts, organic-mineral coordination complexes as well as colloidal particles of amorphous and crystalline structure.

The length of aliphatic chains in the molecular structures of coal can be determined by the diversity of the decomposition processes of the paleomoor mortmass. Anaerobic processes of decay and fermentation can occur in various biochemical ways, depending on temperature, acidity, redox potential, water flow rate and other parameters of the water basin. For example, different types of bacteria as a result of metabolic decomposition of complex organic nutrients produce alcohol, acetic acid, propionic acid, butyrate, lactic acid, butyl-alcohol-acetone fermentation and other fermentations. The resulting products of incomplete decomposition are accumulated in the environment and have in their aliphatic radicals a different ratio of methyl and methylene groups. 
The width of the EPR signal is determined by the quality or diversity of the nature of the PMC. In addition to the free radicals of the organic matrix, paramagnetism in coal can be associated with the presence of transition metals in the molecular structure of paramagnetic ions, primarily iron and the presence of heteroatoms in organic compounds $(\mathrm{O}, \mathrm{N}, \mathrm{S}$ and $\mathrm{P})$. The accumulation of these atoms in the coal structure is due to the geochemical situation of the sedimentation period and the characteristics of the cycle of the corresponding elements. Obviously, this factor does not depend on the decomposition of plant biomass and the introduction of mineral particles.

Summarizing the results obtained, the four identified factors can be reduced to two global phenomena affecting the molecular structure of coal. The main factor, which is two times more significant in terms of its effect on the parameters under study, is a single factor of metamorphism and a subordinate multi-faceted factor of sedimentation conditions.

Since it is not possible to comprehensively characterize the whole diversity of the molecular structure of coal with several parameters, we briefly examine DRIFT of several particular cases of variations in the molecular structure of coal, which are comparable in the degree of metamorphism depending on the formation depth.

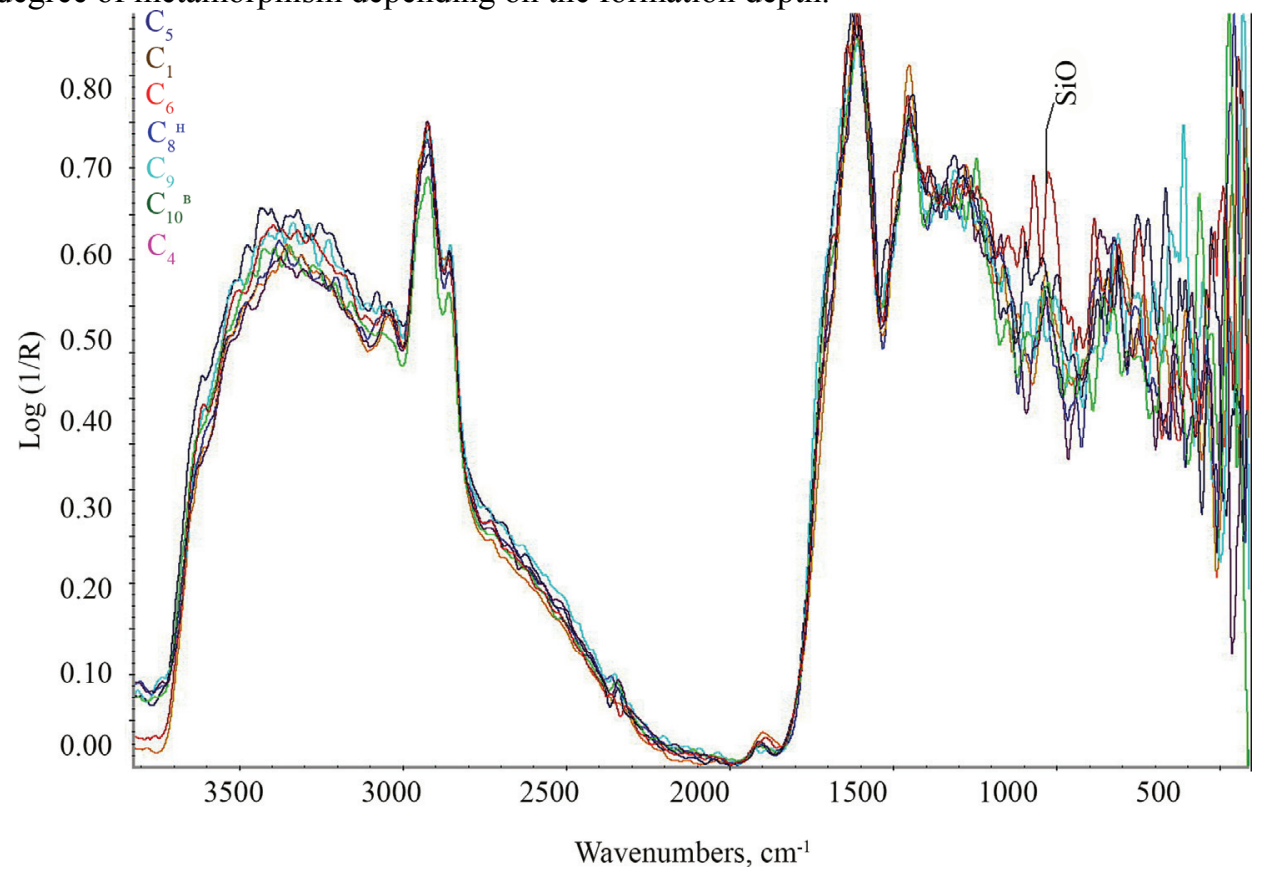

Fig. 2. DRIFT of coal from various beds of lower carbon from holes, mine "Zakhidnyi Donbas".

The slight changes observed in the DRIFT samples of subbituminous carbon from the bore holes, the field of the mine "West-Donbas" (Fig. 2) do not occur symbatically with increasing depth (beds $\mathrm{c}_{1}-\mathrm{c}_{10}$ ). The sample from the $\mathrm{c}_{6}$ formation is separated by the peak of asymmetric valence vibrations of the bridge $\mathrm{Si}-\mathrm{O}-\mathrm{Si}$ group reflecting increased silicate content due to the accumulation of clay minerals during the sedimentation period. In general, the molecular structure of these coals is identical, which indicates similar conditions of sedimentation and subsequent coalification.

The spectra of two samples of subbituminous coal from the $\mathrm{n}_{1}$ seam from different depths of the Butivska mine are also similar in the form (Fig. 3). However, coals from a depth of $1060 \mathrm{~m}(\mathrm{CH} / \mathrm{OH}=5.15 \%)$ can be considered more metamorphic than from the level of $400 \mathrm{~m}(\mathrm{CH} / \mathrm{OH}=4.2 \%)$ due to a decrease in the content of bound hydroxyl groups and a small increase in the concentration of hydrophobic $\mathrm{CH}$ groups. 




Fig. 3. DRIFT of sub-bituminous coal, bed $\mathrm{n}_{1}$ (depth 400 and $1060 \mathrm{~m}$ ) and bed $\mathrm{c}_{1}$.

Immersion at $660 \mathrm{~m}$ of the bed leads to defluidization by leaving water molecules out of the coal substance. In comparison, coals from the bore hole of the mine "Zakhidnyi Donbas" of bed $\mathrm{c}_{1}$, which at the volatile yield formally belong to the same rank on the basis of DRIFT $(\mathrm{CH} / \mathrm{OH}=10.28 \%)$ approach the HVB type.

The strongest differences were recorded in samples from the super-deep bore hole of the Zasyadko mine. The shape of an DRIFT sample of coal from a depth of $1986 \mathrm{~m}$ is characteristic of Medium volatile bituminous and is very different from coal from depths of more than $3 \mathrm{~km}$ (Fig. 4). At the same time coal spectra shapes from depths of 3027, 3116 and $3195 \mathrm{~m}$ are typical of Low volatile bituminous. This is confirmed by the change in the hydrophobicity criterion (Table 3). Within this group of coal the change in the DRIFT is not proportional to their formation depth. The shape of the spectrum of the average sample is slightly more metamorphosed compared to the upper and lower beds. Such a distribution can be influenced by mineral components introduced during formation of the bed during sedimentation.

The difference in depth of more than $1 \mathrm{~km}$ leads to the elongation of averaged aliphatic chains, a significant increase in the proportion of aromatic protons, a reduction in the number of oxygen bridges in the conjugated condensed aromatic structures and a strong increase in the content of free hydroxyls. It can be assumed that the growth of free $\mathrm{OH}$ groups in the process of metamorphism occurs due to the release from the weak H-bond of other hydroxyls of $3544 \mathrm{~cm}^{-1}$ (Fig. 4) and in general the role of the hydrogen bond as one of the mechanisms of intermolecular interaction is leveled.

In total the presence and location of characteristic peaks in the 4-coal samples under study, the change in their ratios corresponds to the influence of the metamorphism factor on mature coals. With high probability one can assume that coal from a depth of $1986 \mathrm{~m}$ with time in the process of immersion can be transformed into one of the coal from depths of more than $3 \mathrm{~km}$, despite permissible differences in sedimentation conditions. 


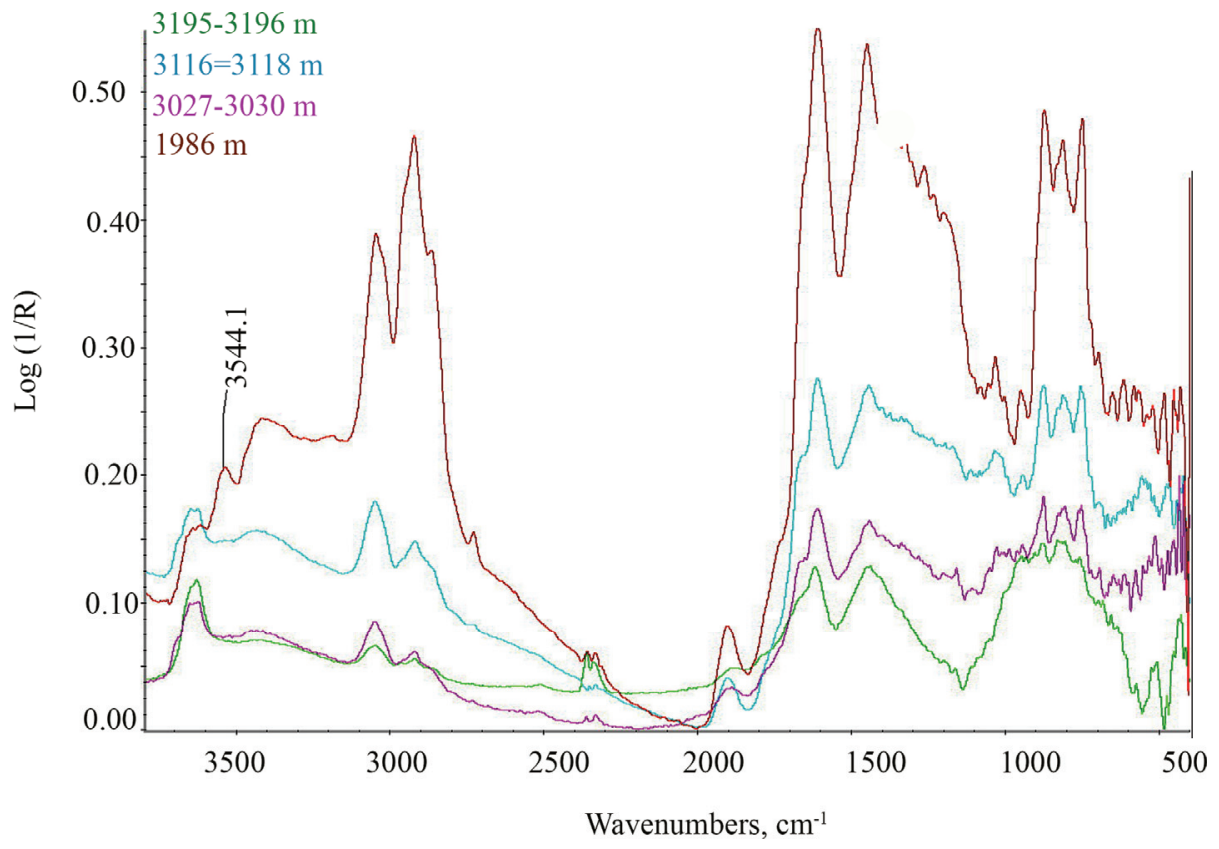

Fig. 4. DRIFT samples of bituminous coals at the different depths of the super-deep bore hole of the Zasyadko mine.

Table 3. The parameters of the molecular structure of coal from the bore hole of the Zasyadko mine.

\begin{tabular}{|c|c|c|c|c|c|c|c|}
\hline Depth, $\mathrm{m}$ & $\mathrm{CH}_{2} / \mathrm{CH}_{3}$ & $\mathrm{CHas} / \mathrm{sym}$ & $\mathrm{CHh} / \mathrm{w}$ & $\mathrm{Har} / \mathrm{Hal}$ & $\mathrm{CH} / \mathrm{OH}$ & $\mathrm{C}=\mathrm{C}-\mathrm{O}$ & $\mathrm{OH}_{\text {free }}$ \\
\hline 1986 & 0.50 & 2.20 & 0.0033 & 0.38 & 0.42 & 1.84 & 1.62 \\
\hline $3027-3030$ & 0.35 & 2.37 & 0.0002 & 1.26 & 0.20 & 0.57 & 3.42 \\
\hline $3116-3118$ & 0.30 & 2.18 & 0.0005 & 1.21 & 0.27 & 1.16 & 3.11 \\
\hline $3195-3196$ & - & 2.14 & - & 1.01 & 0.13 & 0.10 & 4.06 \\
\hline
\end{tabular}

\section{Conclusions}

The research of Donbas coal samples confirmed the general tendency of the influence of the metamorphism factors on the reduction of molecular diversity of oxygen-containing compounds and the shortening of aliphatic chains under the simultaneous increase in the number of paramagnetic centers and conjugated aromatic carbon structures.

The factor analysis by the method of the main components of the molecular structure parameters in coal showed that the most significant independent component is metamorphism, which is a combination of temperature gradients, pressures and various tectonic influences. The remaining subordinate components can be attributed to the conditions of sedimentation, which include independent processes: accumulation of mineral components, decomposition of biomass and geochemical environment in the sedimentation period.

The beds of sub-bituminous coals of early-, middle- and late carboniferous age occurring at shallow depths have insignificant differences in the molecular structure, at the same time, a deep immersion of bituminous coals leads to strong changes in their molecular structure.

The introduced hydrophobicity criterion showed its informativeness in the analysis of the molecular structure of coals. It is more sensitive to changes in the coal rank than the standard index of volatile yield. The coalification process leads to a significant increase in this 
index due to the water release and other hydroxyl-containing compounds from the substance of coal.

\section{References}

1. J.G. Speight, Handbook of Coal Analysis (John Wiley \& Sons, Hoboken, 2005)

2. Xiaoshi Li, Yiwen Ju, Quanlin Hou, Zhuo Li, Junjia Fan, J. Geol. Resear., 8 (2012)

3. He Xueqiu, Liu Xianfeng, Nie Baisheng, Song Dazhao, Fuel, 206, 9 (2017)

4. E.V. Ul'yanova, O.N. Malinnikova, A.V. Burchak, A.K. Balalaev, V.I. Baranovsky, J. Mining Sci., 53, 8 (2018)

5. Jie Wang, Yaqun He, Yong Yang, Weining Xie, Xiangyang Ling, Physicochem. Probl. Miner. Process, 53(1), 13 (2017) 